\title{
¿Por qué plantar en Patagonia? Estado actual y el rol futuro de los bosques plantados
}

\author{
José O. Bava ${ }^{1,2, \varpi}$; Gabriel A. Loguercio ${ }^{1,2} \&$ Gustavo SAlvador ${ }^{1,2}$ \\ 1. Centro de Investigación y Extensión Forestal Andino Patagónico (CIEFAP). 2. Universidad Nacional de la Patagonia "San \\ Juan Bosco" Esquel, Chubut.
}

\begin{abstract}
Resumen. El uso de madera producida de manera sustentable es más amigable para el ambiente que el de materias primas cuya elaboración requiere una proporción elevada de energía fósil. La demanda de madera es creciente, y las plantaciones pueden satisfacerla. En Patagonia se está consolidando un capital importante en bosques plantados, a pesar de los incendios, del retraso en el manejo y del avance de la urbanización sobre las forestaciones. Una concepción económico-financiera del desarrollo indica que las plantaciones de árboles deben restringirse a las zonas con mayor potencial productivo del país y que la Patagonia debería importar madera de esas regiones. Sin embargo, motivos socioeconómicos, productivos y ambientales justifican el fomento de las forestaciones en Patagonia. La demanda regional de madera crece, y para satisfacerla se requieren fletes de más de $2000 \mathrm{~km}$ de distancia, con el consiguiente impacto sobre las emisiones de $\mathrm{CO}_{2}$. Como contracara, es una oportunidad para desarrollar una producción forestal basada sobre empresas locales. La región posee un potencial biofísico para forestaciones y podría satisfacer su demanda ocupando una mínima parte del territorio, en un contexto en que los beneficios de la inversión serán percibidos por la generación siguiente a la que realiza la plantación. Su desarrollo requiere de escasa tecnificación y — por ello- genera más empleo que en otras regiones del país. Las plantaciones pueden moderar o revertir los procesos de deterioro de suelos. Los bosques, manejados de forma adecuada, mantienen parte de la vegetación nativa, la vida silvestre, y pueden capturar grandes cantidades de $\mathrm{CO}_{2}$ adicional. La Patagonia posee un conglomerado de instituciones dedicadas a la ciencia y la tecnología que ha generado las bases para conducir de manera sustentable plantaciones en las que se atiendan las consideraciones de localización, diseño y manejo.
\end{abstract}

[Palabras clave: plantaciones forestales, pino ponderosa, desarrollo forestal]

Aвstract. Why planting trees in Patagonia? Current status and future role of tree plantations: The use of sustainable-produced timber is more friendly with the environment than that of raw materials which require high amounts of fossil energy to be produced. Plantations can satisfy the increasing demand for timber. In Patagonia, an important capital of tree plantations is being consolidated (in spite of forest fires, management lags and urbanization expansion over plantations). Although an economical and financial point of view indicates that plantations should be restricted to areas with the highest productive potential, from which Patagonia should import timber, there are socio-economic, productive and environmental reasons justifying the promotion of plantations in the region. On the one hand, transport across more than $2000 \mathrm{~km}$ is required to satisfy the increasing regional demand of timber (with the consequent impact on $\mathrm{CO}_{2}$.emissions). On the other hand, plantation promotion represents an opportunity for Patagonian forest development based on local companies. The region has a good biophysical potential for tree plantations, and the demand for timber can be met by occupying only a small fraction of the territory, with the investment returns being received by the following generation. Afforestation development requires low mechanization and therefore it creates more jobs than in other regions. Plantations can moderate or even revert degradation and under proper management they can preserve part of the native vegetation and wildlife, capturing large amounts of additional $\mathrm{CO}_{2}$. Patagonia has a major number of institutions devoted to science and technology which have lay the ground for sustainable steering of plantations as long as they display the right location, design and management.

[Keywords: forest plantations, ponderosa pine, forest development]

“Una tarde de otoño subí a la sierra y al sembrador, sembrando, miré risueño; y desde que existen hombres sobre la tierra ¡nunca se ha trabajado con tanto empeño!

Quise saber, curioso, lo que el demente sembraba en la montaña sola y bravía; el infeliz oyó me benignamente y me dijo con honda melancolía:

—Siembro robles y pinos y sicomoros; quiero llenar de frondas esta ladera, quiero que otros disfruten de los tesoros que darán estas plantas cuando yo muera."(...)

Fragmento de "El Sembrador" de Marcos Rafael Blanco Belmonte 


\section{¿Por Qué Producir madera EN BOSQUES PLANTADOS?}

Los árboles son organismos que generan servicios ecosistémicos. Uno de ellos es la producción de madera, una materia prima demandada por la sociedad, cuya producción y uso presenta mínimos impactos colaterales negativos en comparación con otros materiales que podrían sustituirla en algunos procesos productivos (e.g., plástico, hierro y cemento, entre otros). Esto se basa en que, siendo un organismo renovable que fija carbono de la atmósfera utilizando energía solar, el uso sustentable de los bosques -en especial de los plantados- reduce los impactos sobre otros recursos naturales y sobre el consumo de energía fósil (Rodríguez et al. 2014).

La FAO ha previsto un crecimiento del $25 \%$ en la demanda mundial de madera entre 1999 y 2010. Sólo en 2010, la revista anual del mercado de productos forestales de la Comisión Económica de la ONU para Europa (UNECE), indicó que el consumo de productos forestales en general en países desarrollados y en vías de desarrollo aumentó 5.6\% (Barr \& Cossalter 2004; Seppälä 2007; FAIMA 2011). Esta demanda no se puede cubrir con bosque nativo. La proporción de los bosques nativos que tiene aptitud para la producción de madera es limitada, y por sus funciones múltiples está sujeta a otras demandas por parte de la sociedad. Es necesario avanzar de manera gradual en la puesta en producción de los bosques y tierras forestales de manera sustentable, tanto a nivel global como en la Patagonia. De todas maneras, las plantaciones son más eficientes para producir madera que los bosques nativos; el país tiene millones de ha disponibles para este desarrollo y Patagonia, en particular, dispone de cientos de miles.

Es importante también destacar que los recursos forestales y el sector asociado evolucionan, madurando con el tiempo el desarrollo de las políticas y prácticas de manejo forestal que en ellos se aplican. Se han observado a nivel internacional patrones comunes de evolución, como lo explican los modelos de Kimmins (1997) y Gamborg \& Larsen (2003), especialmente en Europa, pero también en otro países como Chile (Bannister \& Pyttel 2010) y Brasil (Machado Leão 2000), entre otros. Éstos describen una evolución que seinicia con la explotación del recurso, pasando por la incorporación de técnicas de manejo y regulaciones de uso, hasta una forestería social y más próxima a la naturaleza, en búsqueda de mayor sustentabilidad. Las plantaciones forestales son parte de esta evolución. En ese contexto, es importante considerar no sólo el estado actual de Patagonia sino el proceso que lo ha caracterizado hasta el presente, así como su posible evolución, considerando las medidas adecuadas para su mejor desarrollo en el largo plazo.

\section{¿DÓNDE ESTAMOS Y CÓMO LLEGAMOS HASTA AQUí?}

Las primeras plantaciones forestales en Patagonia que permitieron identificar aquellas especies exóticas con mejor comportamiento se remontan a principios del siglo XX. Se realizaron en Bariloche, que estaba creciendo como "la Suiza Argentina", de acuerdo a la visión de la época (Bailey-Willis 1914). En 1916 ya funcionaba un vivero con sauces y pinos, administrado por el Ministerio de Agricultura de la Nación (Havrylenko 2005). Luego, la Administración de Parques Nacionales (1934) realizó reforestaciones para la recuperación de bosques nativos muy degradados por incendios y sobrepastoreo en la Isla Victoria, además de crear el vivero homónimo (Municipalidad de San Carlos de Bariloche sf), experiencia que luego se expandió a situaciones similares en El Bolsón y el Parque Nacional Los Alerces (Loguercio et al. 2005a).

A medida que se crean las provincias patagónicas se implementa la ley Nacional de Bosques $N^{\circ} 13273$, de 1945, que promueve el uso de los bosques nativos mediante planes de ordenación y la primera promoción de las forestaciones. Al mismo tiempo se establecen estaciones forestales en El Bolsón y Trevelin, que amplían los ensayos de especies exóticas, con una visión productivista. El objetivo fue promover el desarrollo económico a partir de la creación de fuentes de materia prima de uso industrial, en consonancia con lo que sucedía en otras regiones del mundo con las plantaciones de pinos o eucaliptos en Nueva Zelanda, Chile, Sudáfrica y sur de Brasil, y en el Litoral de nuestro país. El modelo, propuesto como válido para toda Argentina, fue el de plantaciones densas (densidad inicial $2 \times 2 \mathrm{~m}, 2500$ plantas/ha), cuyo manejo produciría madera para la producción de celulosa. Con el transcurso de los años se observó que para la Patagonia este modelo no fue apropiado por las condiciones 
geográficas, ambientales y económicas (grandes distancias entre plantaciones, bajo crecimiento, poca infraestructura). Como resultado, no seinstalaron industrias de síntesis y las plantaciones no fueron adecuadamente manejadas. Esto favoreció la ocurrencia de daños por ataque de Sirex (Carnegie et al. 2006; Villacide et al. 2014) y las pérdidas por incendios en masas sin manejo (Defossé et al. 2001). En las décadas del setenta y ochenta se plantó con el régimen de promoción del Instituto Forestal Nacional (IFONA), y en Patagonia se establecieron plantaciones subsidiadas. En ciertos casos se reemplazaron superficies de bosques nativo (en particular, de Austrocedrus chilensis (D. Don.) Pic. Serm. et Biz. por plantaciones de Pseudotsuga mensiezzi (Mirb) Franco, Pinus radiata D. Don., Pinus contorta subsp. murrayana (Grev. \& Balf.) Critchf. y Pinus ponderosa Douglas ex Lawson) para aprovechar el mayor crecimiento en dichos ambientes. Con el tiempo se fueron consolidando las Direcciones provinciales de Bosques con ingenieros forestales, quienes desarrollaron un conocimiento empírico local que generó ciertos cambios. A partir de 1982 se prohibió la práctica de desmonte pesado y la plantación se restringió a las zonas libres de bosque, disminuyendo las densidades iniciales a $3 \times 3$ (1111 plantas/ha).

Pülzl et al. (2010) proponen que los discursos globales sobre bosques han influido sobre las políticas internacionales y que los cambios discursivos se han reflejado en cambios políticos. Así, el meta discurso neoliberal asociado a una desregulación y autorregulación de leyes blandas (Arts et al. 2010) definió la creación de leyes de fomento forestal cuyos efectos y alcances serían delineados por el mercado. Con este espíritu, a partir de la sanción de la Ley Nacional $\mathrm{N}^{\circ}$ 25080 de Inversiones para Bosques Cultivados (en 1998) se retoma la promoción que se había interrumpido con la disolución del IFONA al inicio de los años 90. En esta ocasión, sólo se subsidiaron plantaciones en el área libre de bosques, una política que se mantiene hasta el presente. En Patagonia, las densidades de plantación se hacen menores, aunque no se explicita con claridad un modelo de desarrollo forestal propio que guíe tanto los objetivos productivos de la plantación como su ubicación y manejo. Las demoras en el pago de los subsidios nacionales y provinciales en muchos casos han provocado que los productores pierdan interés ante lo incierto de este mecanismo, y que empresas de servicios de plantaciones o producción de plantas debieran enfrentar mayores riesgos, inestabilidad y pérdida del apalancamiento financiero.

El resultado de este proceso en Patagonia, es la presencia de plantaciones dispersas desde el norte de la provincia de Neuquén hasta el centro y sur de la zona andina del Chubut. Una parte de la superficie implantada fue consumida por incendios forestales (5600 ha entre 1999 y 2009 [Defossé et al. 2011]). Otra parte importante de las plantaciones no ha sido manejada de forma adecuada, lo que ocasionó problemas sanitarios como la eclosión del Sirex (Villacide \& Corley 2006; Villacide et al. 2014). Además, la expansión de las áreas urbanas y periurbanas también ha afectado a las plantaciones que hoy constituyen barrios residenciales o están en proceso de fraccionamiento. Sin embargo, también hubo casos en que la forestación estimuló el desarrollo de asentamientos poblacionales (e.g., Manzano Amargo, Huinganco, etc.) y a la mejora de la calidad de vida urbana.

El uso histórico y actual dominante del suelo es la ganadería extensiva (ovina, bovina y caprina). El sobrepastoreo ha producido cambios en la estructura y el funcionamiento de las estepas (graminosas a arbustivograminosas, arbustivas y eriales). A su vez, la pérdida de cobertura ha activado procesos de erosión (en algunos sitios muy marcados), lo que produjo la desertificación de parte del territorio (Paruelo et al. 1993; Aguiar et al. 1996; López et al. 2013). En este contexto, los productores han decidido localizar las forestaciones en estos ambientes degradados con escasa oferta de forraje (Loguercio 2015), donde la exclusión del ganado en el cortomediano plazo tiene un bajo impacto en la producción total y en la capacidad de generar ingresos prediales.

En el contexto de un desarrollo que aún no ha llegado masivamente al primer turno de corta, y que se caracteriza porque los beneficios de la inversión serán percibidos por una generación siguiente a la que realiza la plantación, se ha logrado la implantación de unas 97400 ha, creciendo a una tasa de entre 7.5 y $19.5 \mathrm{~m}^{3}$.ha ${ }^{1}$.año ${ }^{-1}$ (Gonda et al. 2009) en Patagonia. La tasa de forestación, que en la región llegó a un máximo de 5000 ha/año durante la década del noventa (Loguercio \& Deccecchis 2006), hoy se mantiene en 1000 ha/año. La superficie 
intervenida con podas y raleos - prácticamente nula hace una década- se ha incrementado y en los últimos cinco años se ha mantenido entre 1000 y 1400 ha/año de poda y raleo en Chubut y Neuquén (SsByP del Chubut, sf, $\mathrm{M}$. Mazzuchelli, comunicación personal). Según el estado de las plantaciones al 2011 se estimó que para el período 2012-2016, a nivel regional unas 4500-5500 ha/año requerían raleos, de las cuales la mitad correspondían a plantaciones jóvenes - que, por lo tanto, también estarían asociados a podas-, y las segundas a raleos comerciales (Loguercio et al. 2011).

Desde el punto de vista industrial, acorde con la llegada de las primeras plantaciones al turno de corta se comienza a invertir en la incorporación de tecnología específica para procesar productos provenientes de los tratamientos intermedios de las plantaciones. Este es el caso de la empresa público-privada Corporación Forestal Neuquina S.A. de Neuquén, y el de muchas Pymes que procesaban materia prima de bosque nativo y han debido reconvertirse para transformar madera de raleos en productos con distinto valor agregado (e.g., madera aserrada verde, machimbre, vigas laminadas, viviendas de madera y muebles macizos). Si bien el impacto de la promoción de las forestaciones no ha sido aún el esperado, ha dado lugar a un capital creciente que demanda empleo en Patagonia y que ofrece productos forestales en una región donde la madera ha sido tradicionalmente incorporada en las construcciones y su uso es alentado por organismos públicos y privados (Hanono 2001). Además, la distancia a los grandes centros de producción de madera supera los $2000 \mathrm{~km}$, lo que genera una oportunidad para satisfacer la demanda regional a partir del desarrollo de empresas locales.

Desde la perspectiva institucional, Patagonia presenta un importante conglomerado de instituciones dedicadas a la ciencia y tecnología que en los últimos 20 años ha generado gran número de investigaciones y publicaciones que sientan las bases del conocimiento para que el manejo de las plantaciones se realice de forma sustentable. Una muestra de esta producción se presenta en la síntesis producida por Gyenge et al. (2010) y en los numerosos aportes para la silvicultura de plantaciones (Andenmatten \& Letourneau 1997, 2003; Broquen et al. 1998; Gonda 2001; Gonda \& Cortés 2001; Davel \& Ortega 2003; Loguercio et al. 2009; Davel 2013; Caballe 2013).

\section{¿HACIA DÓNDE VAMOS?}

El crecimiento económico no se condice con los tiempos biológicos y la capacidad del ambiente para producir bienes y servicios, como tampoco la resiliencia de los sistemas afectados por el mismo (Chapin III et al. 2009). Las consecuencias de este crecimiento sin límites se hacen sentir tanto en lo global como en la región. El cambio climático ha dejado de ser una amenaza futura para instalarse como una realidad que, en general, golpea a los más débiles. En el país, centenares de miles de hectáreas de bosque chaqueño han sido desmontadas en las últimas dos décadas para ser transformadas en cultivos agrícolas, proceso cuyas consecuencias son inciertas (UMSEF 2007, 2012). En Patagonia, la actividad ganadera tradicional ha resultado en graves procesos de desertificación que afectan amplias superficies (León \& Aguiar 1985; INTA-GTZ 1995; del Valle et al. 1997; Golluscio et al. 1998). Una concepción economicista del desarrollo, basada sólo en la maximización del beneficio financiero, indica que la actividad de plantaciones debe restringirse a las zonas de mayor potencial productivo del país, donde los indicadores financieros son mayores y se puede tentar a inversores nacionales e internacionales con una alta tasa de retorno de las inversiones. De acuerdo a esa visión, la Patagonia debería importar madera de esas zonas productivas, beneficiándose de los bajos precios que la escala de producción y los vaivenes de la economía internacional permiten. De hecho, la recomendación del Seminario "El Desafío de las Políticas Forestales en Argentina" (CPIA 2014), organizado por el Consejo de Profesionales de Ingeniería Agronómica y que contó con la presencia de representantes de Federación Argentina de la Industria de la Madera y Afines (FAIMA), de Asociación de Fabricantes de Celulosa y Papel (AFCP), de la Corporación Chilena de la Madera (CORMA), fue la de concentrar la promoción en las zonas de mayor producción (e.g, las provincias del Litoral).Sin embargo, desdeotro punto devista, a nivel global y debido a la gran competencia por el uso del suelo y la necesidad creciente de servicios ecosistémicos, es muy probable que las plantaciones en el futuro sean preferidas en lugares donde más aporten a mejorar los ecosistemas y su funcionamiento en el paisaje, y con ello contribuir a incrementar la provisión de servicios ambientales (Bauhus et al. 2010). Tal es el caso de plantaciones con diseño y manejo adecuado en áreas degradadas que 
previamente han sido sometidas a un uso no sustentable, como sucede en Patagonia. Esta idea podría extenderse también a los servicios que las plantaciones pueden brindar en términos socioeconómicos. Así, consideramos que es necesario fomentar el establecimiento de nuevas plantaciones en Patagonia. ¿Por qué?

\section{¿Cuáles son los motivos SOCIOECONÓMICOS Y PRODUCTIVOS PARA FOMENTAR EL DESARROLLO Y ESTABLECIMIENTO DE LAS PLANTACIONES EN PATAGONIA?}

Desde el punto de vista económico, el aumento de la demanda de madera en la región norpatagónica por el incremento de la población se satisface con madera proveniente de las industrias de la Mesopotamia, lo que apoya la dependencia económica extraregional, con mayores costos locales y beneficios que se exportan a otras regiones. Una estimación realizada en Chubut mostró que en los años 2006-2007 se importó 88\% del machimbre y $71 \%$ de la tirantería consumida en la provincia (Salvador et al. 2007), lo que equivale a $\$ 80$ millones a valores actuales. Es razonable esperar que esta tendencia se mantenga o se incremente con el aumento de la población en Patagonia. Desde el punto de vista energético, utilizando los factores de conversión citados por Castells \& Bordes Alsina (2012), realizar el transporte por camión de un equipo de madera una distancia de $3000 \mathrm{~km}$ casi equivale a consumir $0.12 \mathrm{KW} / \mathrm{h}$ por pie cuadrado de madera, y genera una emisión de $0.15 \mathrm{~kg}$ de $\mathrm{CO}_{2}$ equivalente por pie cuadrado de madera.

En un estudio realizado en la provincia del Chubut, Aguado (2006) indicó que la producción de madera de bosque nativo presentó su máximo en la década de los ochenta, en coincidencia con el mayor incremento poblacional de las ciudades de la provincia ubicadas en la costa del Atlántico. En la actualidad, los bosques nativos no están en condiciones de satisfacer esta demanda de madera en el corto y mediano plazo debido a que los propietarios de la tierra priorizan el uso pastoril de los mismos y las empresas forestales se enfrentan a problemas de acceso a esta materia prima, tanto por la razón ya mencionada como por la necesidad de realizar importantes inversiones en caminos, entre otras causas. A pesar de que las plantaciones de más edad no han recibido el manejo adecuado para producir madera de calidad, localmente ya están empezando a jugar un rol en la oferta (Figura 1). Una estimación regional arrojó que de ponerse bajo manejo forestal las plantaciones existentes se podría esperar un volumen de producción anual máximo cercano a $290000 \mathrm{~m}^{3} /$ año (32\% rollizos y $68 \%$ productos maderables menores), además de $59000 \mathrm{t}$ de residuos por año (Loguercio et al. 2008).

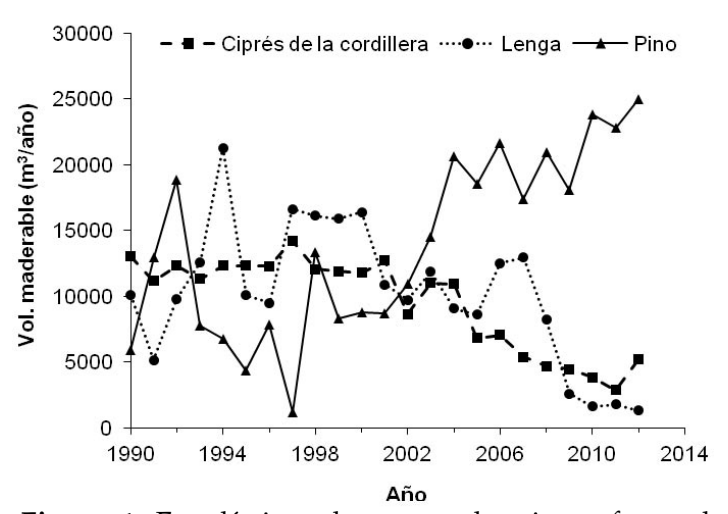

Figura 1. Estadísticas de aprovechamiento forestal de la provincia de Chubut entre 1988 y 2014. Fuente: Subsecretaría de Bosques y Parques del Chubut (no publicado).

Figure 1. Statistics of timber harvested in Chubut province between 1988 and 2014. Source: Chubut forest office (unpublished).

Es importante destacar que aunque la productividad de las plantaciones en Patagonia es muy superior a la de las mismas especies en su lugar de origen (Gonda 1998), los turnos medios para producir madera libre de nudos al turno rondan 43 años (Fernández et al. 2007). Ello implica que los ingresos, que se logran especialmente en las cosechas, beneficiarán a la generación posterior a la que hizo la inversión productiva, aspecto que no puede soslayarse al diseñar las medidas de fomento. La duración de los turnos hace más compleja - y relativiza - la utilización de indicadores financieros de rentabilidad dado que para éstos hay que asumir tasas de descuento del capital (cuya predicción no es realista para períodos tan largos).

En contraposición, se estima que en la región existe un gran potencial biofísico para el desarrollo de forestaciones (CIEFAPFUNDFAEP 2015), con superficies extensas y muy degradadas (Paruelo et al. 1993; del Valle et al. 1997). Desde el punto de vista 
productivo, la existencia de grandes áreas de estepa degradada que no tienen un uso productivo alternativo se puede ver como una oportunidad que podría compensar la menor productividad en relación a otras regiones. En un escenario de planificación racional de forestaciones y una visión de largo plazo, podría considerarse una tasa anual igual al promedio histórico, que ronda las 2500 ha/año (98000 ha/40 años). Para lograr una producción sostenida de madera, ésta debería mantenerse hasta cumplido un turno en cada calidad de sitio (según la productividad del sitio, la edad de cosecha será menor o mayor). El resultado sería una superficie total forestada de 127000 ha adicionales a las existentes. Con este plan, aplicando podas y raleos en el momento adecuado, podría producirse desde el año 20 madera de raleos, que a los 50 años llegaría en forma sostenida a más de 200000 $\mathrm{m}^{3}$ /año (Figura 2). A la cosecha desde el año 40 se obtendría madera de calidad, que a partir de los 60 años mantendría una tasa constante de $980000 \mathrm{~m}^{3}$ / año (Figura 2).

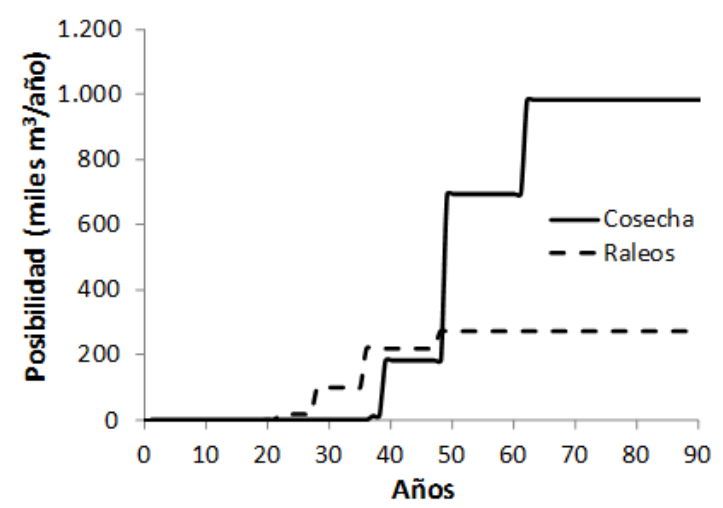

Figura 2. Producción forestal según el desarrollo de un plan estratégico de forestaciones de 2500 ha/año en un tiempo equivalente al turno en cada calidad de sitio.

Figure 2. Forest production by developing a strategic afforestation plan of $2500 \mathrm{ha} / \mathrm{yr}$ in a time equal to the cutting cycle for each site quality.

Por otra parte, el modelo productivo de las plantaciones en Patagonia es muy favorable para la generación de empleo. A diferencia de otras regiones del país y del mundo, la forestación en esta región se caracteriza por su inversión baja en maquinaria y alta en mano de obra. Tanto la producción de plantas en vivero como la actividad de plantación son intensivas en mano de obra. Se requiere personal para la cosecha de semillas, la siembra y las demás labores culturales en vivero e invernáculo, el transporte, la organización, la distribución y la plantación, lo mismo para el control de liebres, la reposición, etc. El raleo pre-comercial, las podas y los raleos comerciales se realizan sin maquinaria pesada (excepto en la provincia de Neuquén, donde la empresa CORFONE S.A. ha incorporado un "harvester"), y sólo se utilizan ciertas máquinas para el arrastre, la carga y el transporte de la materia prima (tractor forestal con pluma). En base a entrevistas a prestadores de servicios y productores de la región, y considerando productividades promedio de la mano de obra, se observa que a lo largo de su desarrollo, las plantaciones generan una demanda creciente de mano de obra (Figura 3). Este factor es mayormente estacional ya que aumenta entre el fin del verano y la primavera, cuando la actividad de las demás producciones de la región es baja; es decir, puede complementarlas sin competir por fuerza laboral. En las zonas con mayor continuidad en forestaciones plurianuales se ha observado la consolidación de asentamientos humanos con relación directa al bosque. Ejemplo de ello son los bosques de asociaciones de fomento rural y los bosques comunales en el norte de la provincia de Neuquén.

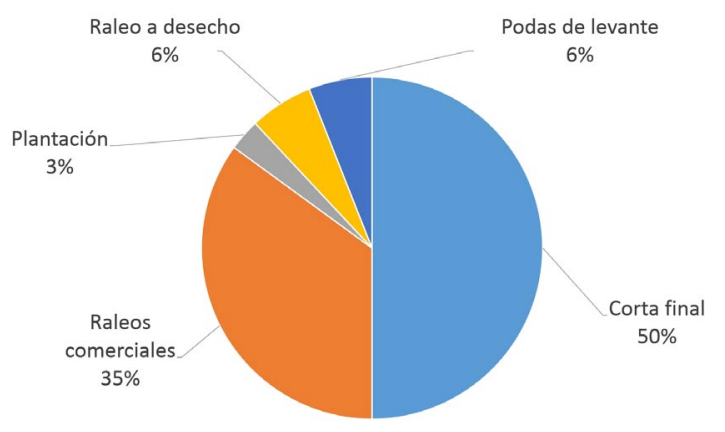

Figura 3. Proporción de mano de obra de las diferentes tareas que demanda la forestación (elaboración propia).

Figure 3. Labor demand by the tasks involved into afforestation.

La madera es la materia prima de innumerables productos, por lo que sufre transformaciones antes de su uso. Esto genera un efecto multiplicador en la generación de empleo a lo largo de sus procesos industriales (Schwarz et al. 2015). Estudios en Argentina muestran que por cada 2 ha plantadas y manejadas se puede generar un puesto directo de trabajo permanente en las etapas de vivero, plantación y cosecha, y se estima que por cada empleo directo se generan entre 0.8 y 1.6 empleos indirectos en servicios de apoyo de silvicultura y de 1.6 a 3 empleos indirectos en la industria (Dalla Tea \& Morales Solís 2005; Schwarz et al. 2015). 
¿Cuáles son las Razones Que JUSTIFICAN FOMENTAR EL DESARROLLO Y ESTABLECIMIENTO DE FORESTACIONES EN PATAGONIA DESDE EL PUNTO DE VISTA AMBIENTAL?

Uno de los principales problemas ambientales del mundo es la pérdida de bosques naturales. Por el contrario, crear nuevos bosques y manejarlos de forma adecuada puede producir beneficios ambientales, además de los antes mencionados. En el caso de la Patagonia, podemos resumirlos en los siguientes aspectos.

1. El uso tradicional de la tierra en las áreas potencialmente aptas para forestar ha causado procesos de degradación de suelos y desertificación en importantes superficies. En esos ambientes, las plantaciones pueden moderar o incluso revertir los procesos de erosión hídrica y eólica (Anderson 1987; Broquen et al. 2003; La Manna et al. 2013, 2014).

2. Los bosques adecuadamente manejados mantienen parte de la vegetación nativa y permiten la presencia de la vida silvestre, proveyendo hábitat para las necesidades alimenticias, de protección y reproducción de diversas especies de animales. Por el contrario cuando no se aplica silvicultura, la competencia por luz, agua y nutrientes, impacta negativamente sobre la vegetación con consecuencias sobre la fauna. Si las forestaciones están diseñadas y manejadas de manera correcta pueden aportar al desarrollo regional sin impactos importantes en la biodiversidad (Gyenge et al. 2010).

3. Otro aspecto ambiental relevante es la posibilidad de capturar grandes cantidades de $\mathrm{CO}_{2}$. La vegetación que tradicionalmente se pastorea en la estepa posee un bajo contenido de carbono, en comparación con la vegetación en otras regiones de mayor productividad. Por ejemplo, en una calidad de sitio media, una plantación de pino ponderosa puede capturar hasta el turno $161 \mathrm{t} /$ ha de C (72\% en la parte aérea y $28 \%$ en las raíces), extrayéndose el $32 \%$ en raleos y permaneciendo hasta la cosecha el $68 \%$ restante (Loguercio et al. 2005b).

4. Las forestaciones establecidas con especies rústicas en ambientes desfavorables, bajo un manejo adecuado, propician un efecto nodriza para la reintroducción de especies forestales nativas bajo la protección de las primeras que, independientemente de que se trate de plantaciones con objetivos de restauración o de producción, podrían tener un rol en disminuir efectos negativos sobre la biodiversidad (Gyenge et al. 2010).

5. La presencia de plantaciones manejadas de forma adecuada en áreas donde antiguamente hubo cierta cobertura de bosques (perdidos por incendios o pastoreo) podría proveer puntos de refugio a especies que podrían conectar pequeños bosquecillos remanentes en el paisaje, en especial si una parte de las forestaciones incluye especies nativas.

6. Se crea un nuevo recurso natural renovable que a través de un manejo sustentable puede mantenerse a perpetuidad. Este recurso puede constituir una reserva que sustituya en el futuro a los recursos no renovables, como el petróleo.

\section{REFLEXIONES FINALES}

Se estima que en la región existe un gran potencial biofísico donde podrían crecer árboles plantados (CIEFAP-FUNDFAEP 2015), buena parte de los cuales se encuentran con signos de degradación severa, como cambios en los tipos fisionómicos de la vegetación, cambios de especies y pérdida de cobertura que han favorecido procesos de erosión del suelo (INTA-GTZ 1995; del Valle et al. 1997; Gaitán et al. 2009). Bajo un escenario de planificación de plantaciones realista y una ordenación forestal que considere una tasa anual igual al promedio histórico (2500 ha/ año [CIEFAP-FUNDFAEP 2015]), la superficie total a forestar, adicional a la existente, ascendería a 127000 ha. Esto podría generar 1250 puestos permanentes directos, 1000 indirectos en tareas silvícolas y 3750 empleos destinados a procesos de transformación de la materia prima. Este desarrollo es compatible con el crecimiento de pequeñas y medianas empresas locales, insertas en las localidades de la cordillera, que se caracterizan por la falta de empleo y / o la alta dependencia del empleo público, y en contados casos, de la demanda estacional del sector turístico.

Respecto a los aspectos ambientales, hay dos rasgos esenciales que merecen ser destacados. Por un lado, el desarrollo posible implicaría la ocupación total de 230000 ha con bosques plantados (97000 existentes más 127000 ha 
nuevas). Éstas representarían entre 4 y $6 \%$ de la superficie libre de bosque considerada como forestable y un porcentaje aún inferior de las estepas graminosas o arbustivas de la Patagonia. Esta proporción es mucho menor que la afectación por forestaciones de la selva misionera o del pastizal correntino. Por otro lado, se trata de plantaciones de turnos largos y densidades menores a las utilizadas en otras regiones, con la ventaja para el ambiente que se realizan sin mediar desmonte ni preparación de suelo ni uso de agroquímicos. En la actualidad, su manejo se está orientando de tal modo de permitir mantener parte de la vegetación original en su interior. Estos dos aspectos son el filtro con el que deben evaluarse la magnitud de los efectos posibles. Cambios en la biodiversidad a escala regional, fragmentación de hábitats o afectaciones del balance hidrológico de humedales no tendrán el impacto que podrían tener en otras regiones o con otro tipo de desarrollo. En casos puntuales, como en la protección de cuencas que aguas abajo presentan ciudades o zonas especialmente degradadas por el uso ganadero, cabría esperar la ocurrencia de efectos positivos por la provisión de servicios ambientales. Debe resaltarse que para morigerar los impactos negativos y potenciar los positivos es esencial que los bosques plantados sean manejados silviculturalmente con pautas con base científico-técnica.

Es necesario continuar con el fomento de las forestaciones por los motivos expuestos. Para ello la pregunta esencial es: ¿qué aspectos es necesario considerar para alcanzar mejores impactos económico-productivos, socioeconómicos y ambientales? En ese sentido, hay que tener consideraciones y cuidados en la localización, el diseño y el manejo de las plantaciones, que podrían resumirse de la siguiente manera:

1. En relación a la localización, es necesario considerar la presencia de sitios especialmente vulnerables (e.g., la proximidad con el bosque nativo), en particular en el caso de relictos de bosque o donde su ubican especies relictuales, humedales, presencia de especies endémicas. En este sentido, puede recurrirse a Rusch et al. (2008), Chehébar et al. (2013) y próximamente a informes de la Unidad para el Cambio Rural del Ministerio de Agricultura, Ganadería y Pesca de la Nación. Desde otro punto de vista, es relevante promover la concentración de bosques plantados en torno a nodos de procesamiento, para aportar al desarrollo de los pueblos y ciudades cordilleranas, considerando también la oferta de tierras en buenas calidades de sitio.

2. En relación al diseño, hay varios aspectos que deben ser considerados. En primer lugar, es necesario plantear la actividad con una mirada estratégica de largo plazo; las plantaciones deben tender a una distribución que cubra en lo posible todo el rango de edades hasta el turno en cada nodo productivo, para evitar que se produzcan discontinuidad o vacíos en el abastecimiento de la industria. Otro aspecto relevante desde el punto de vista ambiental son las consideraciones para lograr la conectividad de las áreas circundantes, en especial donde hubiera especies de especial interés o que se presumiera que pudieran verse afectadas en cada zona. En algunas áreas donde la vegetación original ha sido el bosque nativo (y que no se encuentran en un proceso de recuperación) es recomendable aplicar una política activa de plantación con especies nativas. Por último, parte de las plantaciones pueden plantearse con fines silvopastoriles, integrando la actividad forestadora - que sigue siendo nueva después de más de 40 años-a los sistemas productivos actuales.

3. En relación al manejo, densidades bajas permitirían el mantenimiento de parte de la vegetación nativa y disminuirán posibles efectos sobre el ciclo del agua (Rusch et al. 2008). La implantación de especies adecuadas al sitio, con énfasis en las de mayor valor productivo, y también de especies nativas tanto para la producción, como para la restauración, son elementos que deben ser considerados. Otro aspecto relevante del manejo es el tratamiento de los residuos de las podas y raleos para reducir el riesgo de incendios y plagas.

4. Desde el punto de vista industrial, el incremento de la demanda de productos provenientes de plantaciones y la escasa participación en el mercado de productos elaborados con materia prima de la cordillera generan una oportunidad para el desarrollo foresto-industrial en la Patagonia. Para ello, las industrias de la región cordillerana deberán aumentar su nivel de competitividad y se requerirá de mayores inversiones en tecnología para alcanzar los estándares mínimos de calidad que permitan aumentar su cuota de participación en el mercado, siendo el costo del flete una importante ventaja comparativa. 
5. Desde la perspectiva socioeconómica se deberá promover el bienestar de los trabajadores a través de la implementación de buenas prácticas de manejo, y el cumplimiento de estándares de seguridad e higiene laboral, orientando a las empresas a la generación de empleo formal. Para ello el estado debe promover políticas de largo plazo de desarrollo y consolidación del sector, fortaleciendo las empresas prestadoras de bienes y servicios y asegurando que los beneficiarios finales de las políticas de fomento sean los pequeños y medianos productores, y las comunidades de su zona de influencia.

6. Para revertir la tendencia actual y volver a ganar la confianza del sector productivo no sólo se deberá asegurar el pago en término de los aportes no reintegrables de las leyes de fomento vigentes para permitir el apalancamiento financiero de las empresas, sino que también se requerirá implementar políticas de desarrollo, que aborden la complejidad del sector desde una visión territorial.

En la Región Patagónica existe el potencial para el desarrollo de forestaciones que puedan generar múltiples beneficios desde el punto de vista económico y social con bajo nivel de impacto sobre el ambiente si se realizan con parámetros técnicos adecuados, e incluso se generarían externalidades positivas. En un contexto político donde faltan alternativas productivas de bajo impacto que permitan mejorar o mantener el estado de bienestar de las comunidades, las forestaciones aparecen como una alternativa viable que podría complementar y fortalecer las economías regionales, en la que el beneficio económico será usufructuado por la siguiente generación y el costo debe ser erogado por la generación actual.

\section{BIBLIOGRAFÍA}

AguAdo, G. 2006. Recopilación histórica de aprovechamientos forestales de especies nativas del Chubut, discriminado según las cuencas forestales de la provincia. Tesis Ingeniería Forestal UNPSJB. Pp. 84.

Aguiar, MR; JM Paruelo; OE Sala \& WL Lauenroth. 1996. Ecosystem responses to changes in plant functional types: an example from the Patagonian steppe. Journal of Vegetation Science, 7:381-390.

Andenmatten, E \& F Letourneau. 1997. Curvas de índice de sitio para Pinus ponderosa (Dougl.) Law de aplicación en la región Andino Patagónica de Chubut y Río Negro, Argentina. Bosque, 18(2):13-18.

Andenmatten, E \& F Letourneau. 2003. Predicción y proyección del rendimiento de pino ponderosa en las provincias de Chubut y Río Negro, Argentina. Revista de Ciencias Forestales: Quebracho, 10:14-25.

ANDERSON, D. 1987. Economic aspects of afforestation and soil conservation projects. The Annals of Regional Science, 21(3):100-110.

Arts, B; M Appelstrand; D Kleinschmit; H Pülzl \& IG Visseren-Hamakers. 2010. Discourses, actors and instruments in international Forest governance. En: Rayner, J; A Buck \& P Katila (eds.). 2010. Embracing complexity: Meeting the challenges of international forest governance. A global assessment report. Prepared by the Global Forest Expert Panel on the International Forest Regime. IUFRO World Series Volume 28. Vienna. Pp. 172.

BAnNister, J \& P Pyttel. 2010. Silvicultura cercana a lo natural (SCN): ¿Una nueva visión para el sector forestal chileno.http://revista.bosquenativo.cl/volumenes/46/ cronica.htm

Barr, C \& C Cossalter. 2004. Chinas development of plantation-based wood pulp industry: government policies, financial incentives and investment trends. International Forestry Review, 6:3-4.

Bauhus, J; B PoKorny; PJ van der Meer; PJ Kanowski \& M KANNINEN. 2010. Ecosistem goods and services - the key for sustainable plantations. En: Ecosystem Goods and Services from Plantation Forests. CIFOR. The Earthscan Forest Library. Pp. 245.

Broquen, P; JL Girardin; G Falbo \& O Álvarez. 1998. Modelos predictores de índice de sitio en Pinus ponderosa Dougl. en base a características del suelo andinopatagónico oriental, $37^{\circ}-41^{\circ} \mathrm{S}$, República Argentina. Revista Bosque, 19(1):71-79.

Broquen, P; G Falbo; A Apcarián; F Canda; J Girardin; et AL. 2003. Relaciones entre las forestaciones, la erosión del suelo y la potencialidad productiva en la transición bosque-estepa (Andinopatagonia, Argentina). Invest. Agrar.: Sist. Recur. Forst., 12(2):99-110.

Caballé, G. 2013. Efecto interactivo de la defoliación del estrato herbáceo y la cobertura del estrato arbóreo sobre el crecimiento del estrato herbáceo en sistemas silvopastoriles. Tesis Doctoral. Escuela Para Graduados «Alberto Soriano»-FAUBA. Pp. 130.

Carnegie, AJ; M Matsuki; DA Haugen; BP Hurley; R Ahumada; et Al. 2006. Predicting the potential distribution of Sirex noctilio (Hymenoptera: Siricidae), a significant exotic pest of Pinus plantations. Ann. For. Sci. 63:119-128 119 (C) INRA, EDP Sciences, 2006 DOI: 10.1051/ forest:2005.

Castells, XE \& A Bordes Alsina. 2012. Energía, Agua, Medioambiente, Territorialidad y Sostenibilidad. Ediciones Díaz de Santos S.A. Madrid. ISBN 978-84-9969-185-1.

CHAPIN III, FS; GP KofinAS \& C FolKe. 2009. A Framework for Understanding Change. Cap. I. En: Principles of Ecosystem Stewardship: Resilience-Based Natural Resource Management in a Changing World. Springer Science+Business Media, LLC 2009. Pp. 387.

Chehébar, C; A Novaro; G Iglesias; S Walker; M Funes; ET AL. 2013. Identificación de áreas de importancia para la biodiversidad en la estepa y el monte de Patagonia. Pp. 112.

CIEFAP-FUNDFAEP. 2015. Evaluaciones Ambientales Estratégicas y Programa de Monitoreo de la Biodiversidad en las plantaciones de la región Patagonia Andina. UCAR. Proyecto de Conservación de la Biodiversidad en Paisajes Productivos Forestales. GEF TF 090118. Informe inédito. 
Dalla Tea, F \& J Morales Solís. 2005. Demanda de mano de obra y su incidencia en los costos silvícolas en el proyecto de Forestal Argentina. Forestal Argentina S.A.

DAvel M. 2013. Poda en plantaciones de Pseudotsuga menziesii en la Patagonia andina, Argentina. Revista Bosque, 34(2):181-189.

Davel, M \& A Ortega. 2003. Productividad por zonas de crecimiento para pino oregón (Pseudotsuga menziesii (Mirb.) Franco) en la Patagonia Andina Argentina. Inves. Agr.: Sist. Recur. For. 12(3).

Defossé, GE; G Loguercio; FJ Oddi; JC Molina \& PD Kraus. 2011. Potential CO2 emissions mitigation through forest prescribed burning: A case study in Patagonia, Argentina. Forest Ecology and Management, 261:2243-2254.

DEl VAlle, HF; NO Elissalde; DA Gagliardini \& J Milovich. 1997. Distribución y cartografía de la desertificación en la región de Patagonia. RIA, 28:1-24.

FAIMA. 2011. Crece uso de la madera como combustible. En: Revista Vetas, http://www.vetas.com/sp/ notas.cgi?NOTA=1259.

Fernández, MV; GA Loguercio; M Ruiz Tagle-Molina \& S HAVRYlenKo. 2007. Modelo de planificación estratégica para la generación de cuencas de forestación en Patagonia. Pág. 133-144 en: Acta de Ecoforestar 2007: "Primera reunión sobre forestación en Patagonia". Esquel, Chubut.

FreER-SMith, PH; MSJ BroAdmeadOW \& JM LynCH (EDS.). 2007. Forestry and Climate Change. CAB International. Wallingford, Oxon. Pp. 253.

GAITÁN, JJ; CR LÓPEZ \& D BRAND. 2009. Efectos del pastoreo sobre el suelo y la vegetación en la estepa patagónica. CI Suelos (Argentina), 27(2):261-270.

Gamborg, C \& JB Larsen. 2003. "Back to Nature" - A sustainable future for forestry. Forest Ecology and Management, 179:559-571.

Golluscio, RA; VA Deregibus \& JM Paruelo. 1998. Sustainability and range management in the Patagonian steppes. Ecología Austral, 8:265-284.

GONDA, HE. 1998. Height-diameter and volume equations, growth intercept and needle length site quality indicators, and yield equations for young ponderosa pine plantations in Neuquén, Patagonia, Argentina. Doctoral Dissertation. College of Forestry, Forest Resources Department. Oregon State University. Pp. 198.

GondA, HE. 2001. Manejo de pino ponderosa. Modelo preliminar para plantaciones en sitios de calidad media en la Patagonia andina. Patagonia Forestal, VII(3):7-10.

Gonda, HE \& G CoRTés. 2001. Ecuaciones para el manejo de las plantaciones de Pino ponderosa en Neuquén. Publicación técnica N ${ }^{\circ}$ 30. CIEFAP-Proyecto Forestal de Desarrollo. SAGPyA. Pp. 24.

GondA, H; J BAvA; G Cortés \& G Loguercio. 2009. Growth of ponderosa pine in the northern patagonian Andes, Argentina. Sesión: Mantenimiento e incremento de la capacidad productiva de los bosques. En: Acta trabajo completo. CFM 2009, XIII Congreso Forestal Mundial. Buenos Aires.

Gyenge, JE; ME Fernández; V Rusch; MM SARAsOla \& TM SCHLichter. 2010. Towards Sustainable Forestry Development in Patagonia: Truths and Myths of Environmental Impacts of Plantations with Fastgrowing conifers. The Americas Journal of Plant Science and Biotechnology, 9-22.

Hanono, M. 2001. Construcción en madera. Producciones gráficas y editoriales Cima. ISBN 987-97545-1-4.
Bariloche, Argentina. Pp. 155.

Havrylenko, S. 2005. Vivero Isla Victoria (1902-1970). Patagonia Forestal Año XI(3):14-16.

INTA-GTZ. 1995. Lucha contra la desertificación en la Patagonia a través de un sistema de monitoreo ecológico. LUDEPA-SME. Pp. 182.

Kimmins, JP. 1997. Forest Ecology. A Foundation for Sustainable Management. $2^{\text {nd }}$ Ed. Prentice Hall. Pp. 596.

La Manna, L; C Buduba \& GA Loguercio. 2014. Actividad forestal en Patagonia: interacciones con propiedades edáficas. XXIV Congreso Argentino de Ciencias del Suelo y II reunión Nacional "Materia orgánica y sustancias húmicas". Bahía Blanca.

La Manna, L; M Rostagno \& C Buduba. 2013. Efecto de las Plantaciones en el control de la erosión en ambientes degradados de la Región Andino Patagónica. Tercer Congreso Latinoamericano IUFRO. San José, Costa Rica.

LEÓN, RJC \& MR AGUIAR. 1985. El deterioro por uso pastoril de estepas herbáceas patagónicas. Phytocoenología, 13: 181-196.

Loguercio, GA; C Buduba \& L La Manna. 2005a. Estación Forestal Puerto Limonao, en el recuerdo de Don Julio Oszust. Patagonia Forestal, XI(1):5-6.

Loguercio, GA; G SAlvador \& S Antequera. 2005b. Estimación preliminar de producción y rentabilidad de certificados de reducción de emisiones temporales en forestaciones de pino ponderosa en la Patagonia. Presentación oral en $3^{\circ}$ Congreso Forestal Argentino y Latinoamericano. En: Acta trabajo completo. Corrientes. Pp. 11.

Loguercio, GA \& F Deccechis. 2006. Forestaciones en la Patagonia Andina: potencial y desarrollo alcanzado. Patagonia Forestal, XII(1):4-6 y XII(2):4-8.

Loguercio, GA; A Jovanovski (ex-aequo); JC Molina \& P Pantaenius. 2008. Residuos de biomasa de forestaciones $y$ aserraderos de la región andina de las Provincias de Neuquén y Chubut. Evaluación preliminar de oferta. JICA-INTASAyDS-CIEFAP. Publicación técnica del CIEFAP N ${ }^{\circ}$ 34. Pp. 79.

Loguercio, GA; JD Lencinas; C Buduba \& J IrISARRI. 2009. Evaluación de la calidad de sitio para planificar forestaciones de pino ponderosa en Chubut. En: Actas de $1^{\circ}$ Reunión sobre Planificación y Legislación Forestal de la Patagonia. Esquel. Chubut. 22-23 de Abril de 2009.

Loguercio, GA; H Gonda \& A Jovanovski. 2011. Necesidades de manejo de los bosques plantados en las provincias de Neuquén, Río Negro y Chubut. Producción Forestal, 1:13-17.

Loguercio, GA; C Buduba; E OYharçabal; H Gonda; D MOHR Bell; ET AL. 2015. Productividad forestal y bases para el desarrollo de sistemas silvopastoriles con pino ponderosa al sur de la provincia del Chubut. PIA 10093. BIRF 7520 AR, UCAR-MINAGRI. Pp. 73.

LÓPEZ, DR; MA BRIZUELA; P WILLEMN; MR AGUIER; G SIFFredi; ET AL. 2013. Linking ecosystem resistance, resilience, and stability in steppes of North Patagonia. Ecological Indicators, 24:1-11.

Machado LeÂo, R. 2000. A Floresta e o homem. Sao Paulo: Editora da Universidade de SaoPaulo: Instituto de Pesquisas e Estudos Florestais. Pp. 448.

Municipalidad de SAn Carlos de Bariloche S.F. Isla Victoria. Datos Históricos. Informe inédito. Pp. 3.

Paruelo, JM; MB Bertiller; TM SChlichter \& FR Coronato. 1993. Secuencia de deterioro en distintos ambientes 
patagónicos: Su caracterización mediante modelos de estados y transiciones. Convenio Argentino-Alemán, Cooperación técnica INTA-GTZ. Pp. 110.

PüLzL, H. 2010. Die Politik des Waldes. Böhlau Verlag Wien. Pp. 249.

Rodríguez, LCE; M Pasalodos-Tato; L Díaz-Balteiro \& JP Mc TAGUe. 2014. The importance of industrial forest plantations. En: Borges, JG et al. (eds.). The Management of Industrial Forest Plantations: Theoretical Foundations and Applications. Managing Forest Ecosystems 33, DOI 10.1007/978-94-017-8899-1_1,SpringerScience+Business Media Dordrecht 2014.

Rusch, V; A Vila \& B Marqués. 2008. Conservación de la biodiversidad en sistemas productivos. Forestaciones del Noroeste de la Patagonia. 1a ed. EEA INTA Bariloche.

SALVADOR, G; MV FernÁNDEZ \& G Robles. 2007. Mercado de Madera para la Construcción proveniente de Plantaciones. Estudio Exploratorio en la Provincia del Chubut. Actas de I Reunión sobre Forestación en la Patagonia (Eco Forestar). Esquel, Chubut, Argentina. 25-27 Abril de 2007.

Schwarz, AG; C Burg \& J Cuevas. 2015. Impacto de los Bosques de Cultivo. Importancia Socioeconómica y efecto multiplicador. Documento de Investigación. Año 34 Edición $N^{\circ}$ 60. Fundación Mediterránea. Pp. 41.

SEPPÄLÄ, R. 2007. Global forest sector: trends, treats and opportunities. En: Freer-Smith, PH; MSJ Broadmeadow \& JM Lynch (eds.). Forestry and Climate Change. CAB
International. Pp 25-30.

Subsecretaría de Bosques y Parques del Chubut. (sf). Estadísticas 1988-2013. Dirección de promoción forestal. Estadísticas no publicadas.

UMSEF (Unidad de Manejo del Sistema de Evaluación FORESTAL). 2007. Informe sobre deforestación en Argentina. Pp. 10, http://www.ambiente.gov.ar/archivos/web/ UMSEF/File/deforestacin_argentina_v2.pdf

UMSEF (Unidad de Manejo del Sistema de Evaluación FORESTAL). 2012. Monitoreo de la Superficie de Bosque Nativo de la República Argentina. Período 2006-2011. Regiones Forestales Parque Chaqueño, Selva Misionera y Selva Tucumano Boliviana. Pp. 61. Disponible en http: //obio.ambiente.gob.ar/multimedia/files/Monitoreo $\% 20$ de $\% 201$ a $\% 20$ superficie $\% 20$ de $\% 20$ bosque $\% 20$ nativ o\%20de\%20la\%20Republica\%20Argentina.pdf.

Villacide, JM \& JC Corley. 2006. Control de niveles de poblaciones endémicos de la avispa de los pinos Sirex noctilio (Hymenoptera siridae) mediante raleo sanitario de hospederos atacados. RIA, 35(1):121-134.

Villacide, JM; M Masciocchi \& JC CoRley. 2014. Avispas exóticas en la Patagonia: la importancia de la ecología de invasiones en el manejo de plagas. Ecol. Austral, 24(2):154-161.

Willis, B. 1914. El Norte de la Patagonia: Naturaleza y Riqueza. Tomo 1. Comisión de Estudios hidrológicos. Ministerio de Obras Públicas. República Argentina. Pp. 473. 